\title{
Correção cirúrgica da esotropia progiressiva do alto míope pela técnica de Yamada: relato de dois casos
}

\author{
Surgical correction of progressive high myopic esotropia by Yamada's technique: \\ report of two cases
}

Fábio Ejzenbaum ${ }^{1}$ Mauro Goldchmit ${ }^{2}$ Carlos R. Souza-Dias ${ }^{3}$

\section{RESUMO}

A "esotropia fixa progressiva" se apresenta como um quadro de esotropia progressiva, em adultos altos míopes, que aumenta até que os olhos fiquem fixos numa esotropia de grande ângulo, geralmente associada a hipotropia, e que tem fracos resultados cirúrgicos. O objetivo deste trabalho é descrever a abordagem cirúrgica de 2 pacientes com "esotropia fixa adquirida progressiva" que foram operados segundo a técnica de Yamada (hemitransposição dos retos lateral e superior), e obtiveram bom resultado pós-operatório.

Descritores: Miopia/complicações; Esotropia/etiologia; Esotropia/cirurgia; Relato de caso

\section{INTRODUÇ̃̃̃O}

A avaliação refrativa dos pacientes estrábicos é de fundamental importância diagnóstica e terapêutica.

Entre os portadores de esotropia, a presença de miopia varia entre $3 \%$ e $8 \%{ }^{(1-5)}$.

Outra entidade pertencente ao capítulo de estrabismo nos altos míopes é a "esotropia maligna" ou "esotropia fixa progressiva adquirida"(6), em que adultos altos míopes de longa duração começam a desenvolver esotropia progressiva, de início tardio, que aumenta até que os olhos fiquem fixos numa esotropia de grande ângulo, geralmente associada a hipotropia. Usualmente são bilaterais, a abdução e a elevação se encontram severamente reduzidas, e os resultados cirúrgicos são pobres, com boa correção cirúrgica no período pós-operatório imediato, mas evoluindo novamente com instalação progressiva de esotropia de grande ângulo.

Em 1997, foi realizado estudo ${ }^{(7)}$ através de ressonância magnética, com pacientes com miopia acima 15 dioptrias esféricas e encontrou-se direcionamento anômalo dos retos lateral e superior. Os autores sugeriram que esse achado ocorreria devido à ectasia escleral temporal superior; dessa maneira, o reto lateral assumiria deslocamento para baixo, e o superior para a região medial (Figura 1), teoria que acreditamos ser mais plausível. Posteriormente, outros estudos vieram confirmar esses achados ${ }^{(8-10)}$. Para resolver a hipotropia, foi realizada a fixação escleral do reto lateral na posição que seria normal, com sutura não absorvível, com técnica parecida a "fadenoperation", obtendo bons resultados ${ }^{(7)}$.

Posteriormente, Yamada et al. descreveram uma técnica cirúrgica eficaz para esses casos aplicada numa paciente com esotropia e hipotropia. Foi realizada hemitransposição da metade lateral do reto superior (RS) e superior do reto lateral (RL) com fixação escleral dos feixes transpostos a $7 \mathrm{~mm}$ 


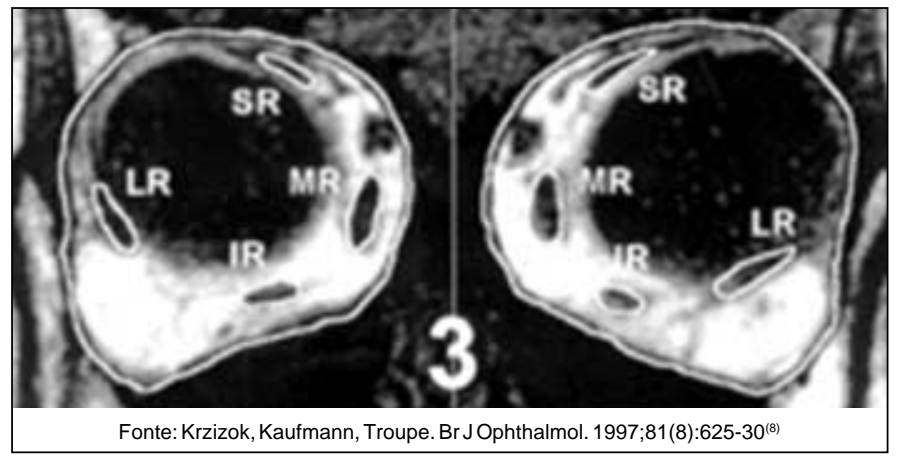

Figura 1 - Corte de ressonância magnética de paciente alto míope com o reto lateral deslocado para baixo

do limbo, associada a retrocesso do reto medial, com a finalidade de obter normalização do trajeto muscular ${ }^{(11)}$.

A seguir, descrevemos dois casos de esotropia associada a hipotropia em pacientes portadores de esotropia progressiva do alto míope, operados na Irmandade da Santa Casa de Misericórdia de SP com a técnica de Yamada.

\section{RELATO DOS CASOS}

\section{Caso 1}

Paciente feminina com 60 anos de idade, míope de -9 dioptrias esféricas (DE) em ambos os olhos (acuidade visual de 0,7 no olho esquerdo), com ambliopia no olho direito (acuidade visual de conta dedos a 1 metro); referia esotropia progressiva desde os 50 anos de idade. Apresentava em posição primária do olhar, ET 50 $0^{\Delta}$ HT E/D $30^{\Delta}$ (Figura 2).

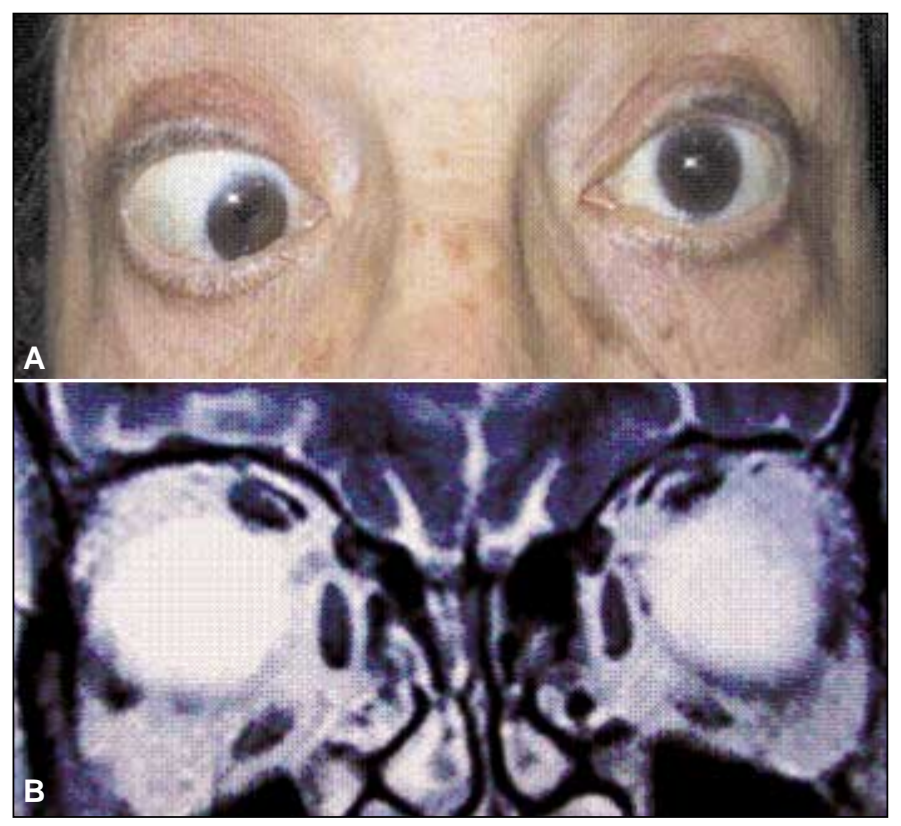

Figura 2 - A: Posição do desvio em PPO; B: Na RNM posição anômala dos retos lateral e superior do olho direito
Ao exame de ressonância nuclear magnética (RNM), era evidente a posição anormal dos retos lateral e superior do olho direito, que se apresentavam deslocados para baixo e medialmente respectivamente (Figura 2).

As fibras dos músculos retos lateral e superior, foram separadas longitudinalmente pela metade, numa extensão de $15 \mathrm{~mm}$. As faixas lateral do RS e superior do RL foram transpostas superior e lateralmente respectivamente, e reinseridas juntas, a meio caminho entre as suas respectivas inserções originais, a $7 \mathrm{~mm}$ de distância do limbo. A faixa do RL foi ressecada (encurtada) $5 \mathrm{~mm}$ (Figura 3). Foi associado também retrocesso do reto medial de $6 \mathrm{~mm}$.

No período pós-operatório imediato, a paciente estava ortotrópica, porém, no décimo dia pós-operatório, houve reincidência da hipotropia. Numa abordagem cirúrgica posterior, encontrou-se a faixa transposta do RL a $5 \mathrm{~mm}$ do local onde fora inserida. Foi realizado o pregueamento de ambos os faixas transpostas dos RS e RL de aproximadamente $3 \mathrm{~mm}$, que foram reinseridas a $7 \mathrm{~mm}$ do limbo. No período pós-operatório, não se notava mais a hipotropia (Figura 4).
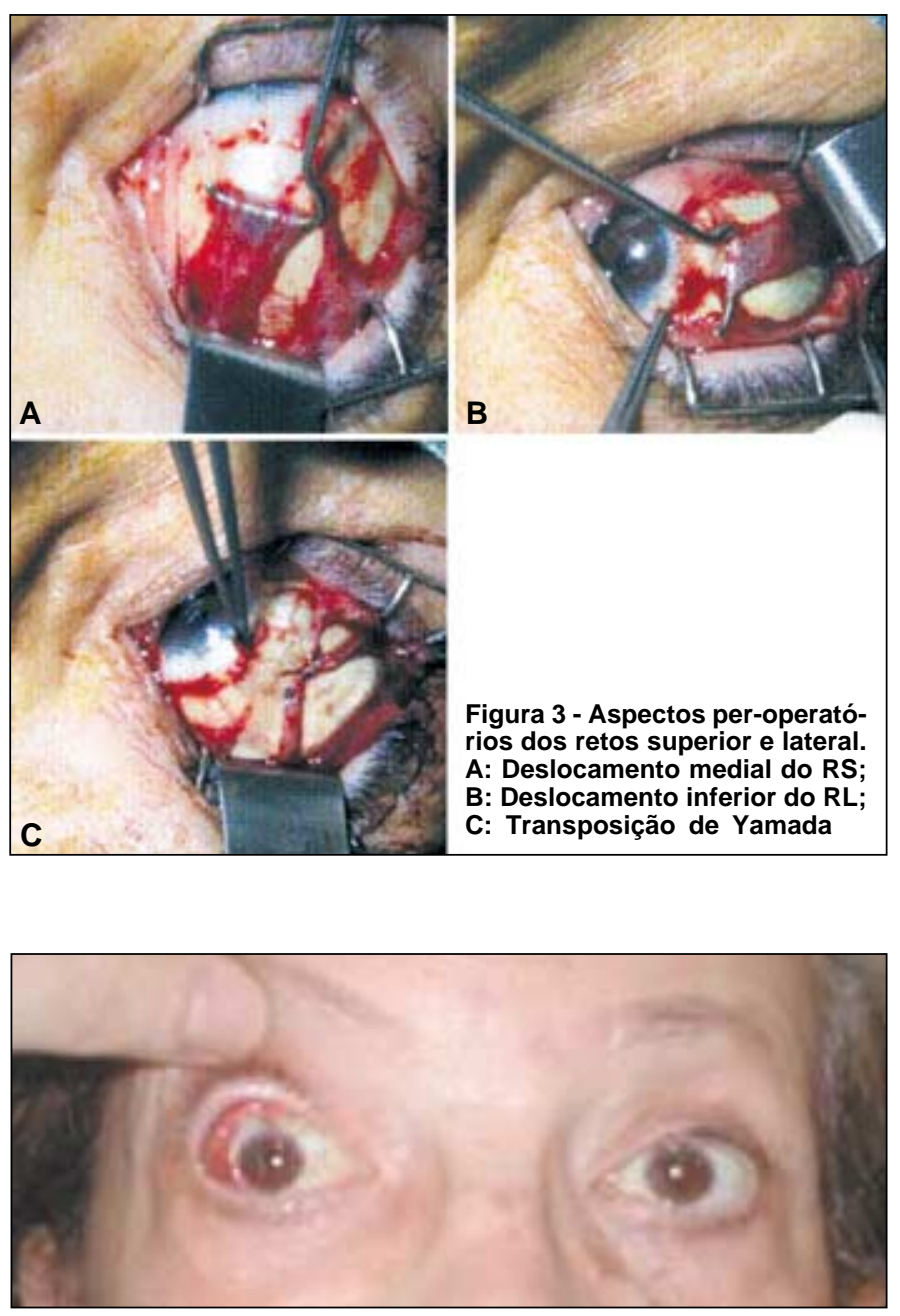

Figura 4 - Pós-operatório da 2a cirurgia 


\section{Caso 2}

Paciente masculino com 50 anos de idade (Figura 5), alto míope em ambos os olhos (-9 DE; acuidade visual de 0,1 no olho direito e 0,6 no olho esquerdo); referia esotropia progressiva desde os 40 anos de idade. Apresentava em posição primária do olhar, ET $60^{\Delta} \mathrm{HT}$ E/D $20^{\Delta}$ e limitação importante de abdução do olho direito (-4).

Embora não tenha sido realizada a RNM antes da cirurgia, foi observado no período per-operatório trajeto anômalo dos músculos retos lateral (Figura 6) e superior.

As fibras dos músculos retos lateral e superior do olho direito foram separadas longitudinalmente pela metade, numa extensão de $15 \mathrm{~mm}$. As faixas lateral do RS e superior do RL foram ressecadas (encurtadas) $4 \mathrm{~mm}$ e transpostas superior e lateralmente respectivamente, e reinseridas juntas, a meio caminho entre as suas respectivas inserções originais, a $7 \mathrm{~mm}$ de distância do limbo. Associou-se ao procedimento o retrocesso do músculo reto medial direito de $6 \mathrm{~mm}$.

No período pós-operatório o paciente encontrava-se em ortotropia (Figura 7).
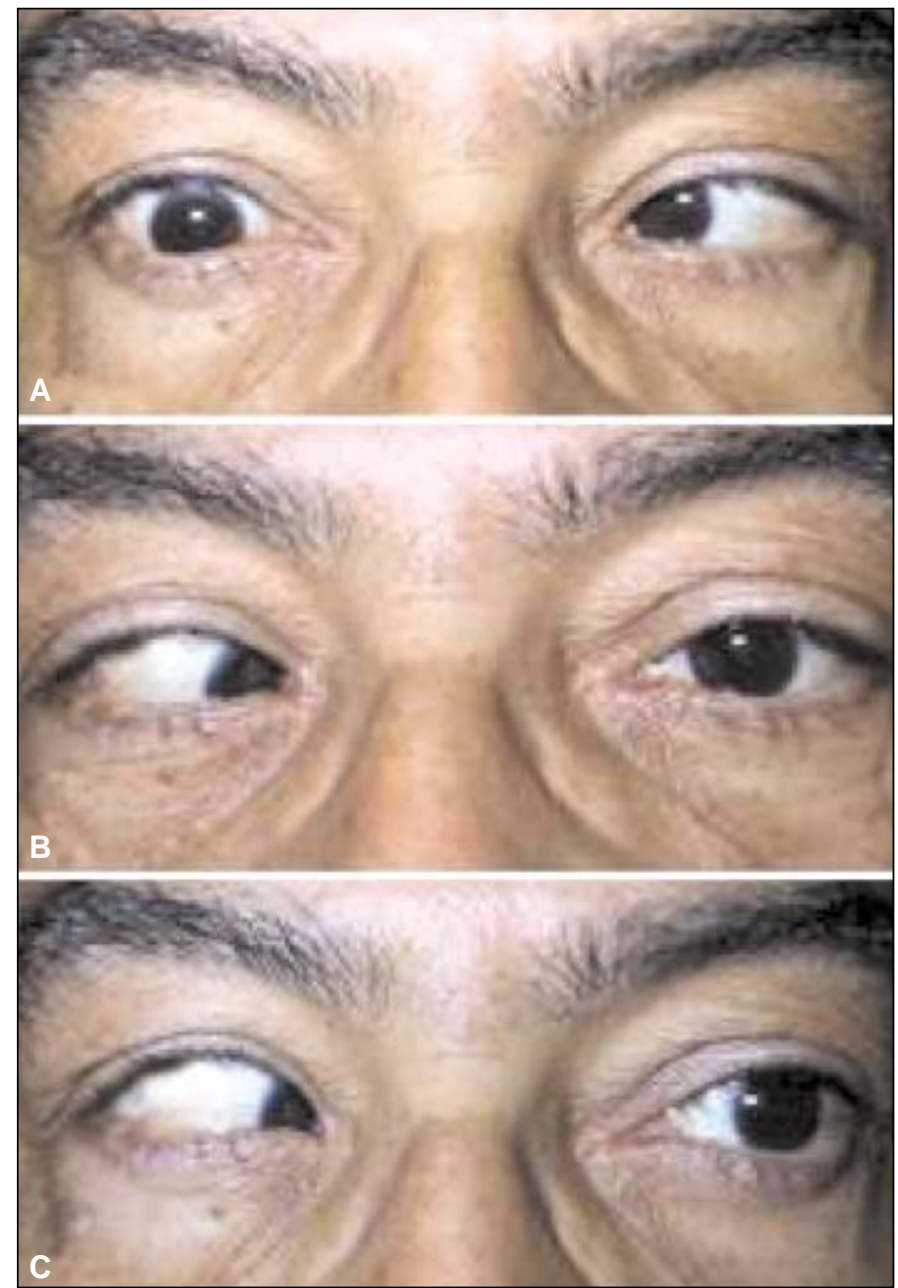

Figura 5 - A: Posição dos olhos em dextroversão; B: PPO e C: levoversão

DISCUSSÃO

Os casos são bastante ilustrativos quanto ao quadro clínico característico da esotropia progressiva do alto míope, com esotropia de início tardio associada a hipotropia e limitação importante da abdução.

Nos dois casos é evidente o deslocamento patológico dos músculos retos lateral e superior, seja no exame de imagem ou no período per-operatório. Esse deslocamento foi primeiramente observado no período per-operatório por outros autores ${ }^{(10)}$.

Segundo outro trabalho ${ }^{(8)}$, que realizou estudo através de ressonância nuclear magnética (RNM), foi observado deslocamento do RL de até $20^{\circ}$ de sua posição original; a medialização do trajeto do RS, causaria redução de sua capacidade supraversora e incremento da adutora, bem como o deslocamento para baixo do RL, seria o responsável pela redução de sua ação abdutora e importante aumento da ação infraversora, levando à instalação da esotropia associada a hipotropia. Com a normalização do trajeto, esses músculos voltariam a ter sua ação original. Porém no presente estudo não podemos afirmar que houve a normalização, pois não possuímos a RNM após a cirurgia.

Acreditamos que no primeiro caso, as fibras transpostas do RL não se encontravam no local inserido, devido à utilização de

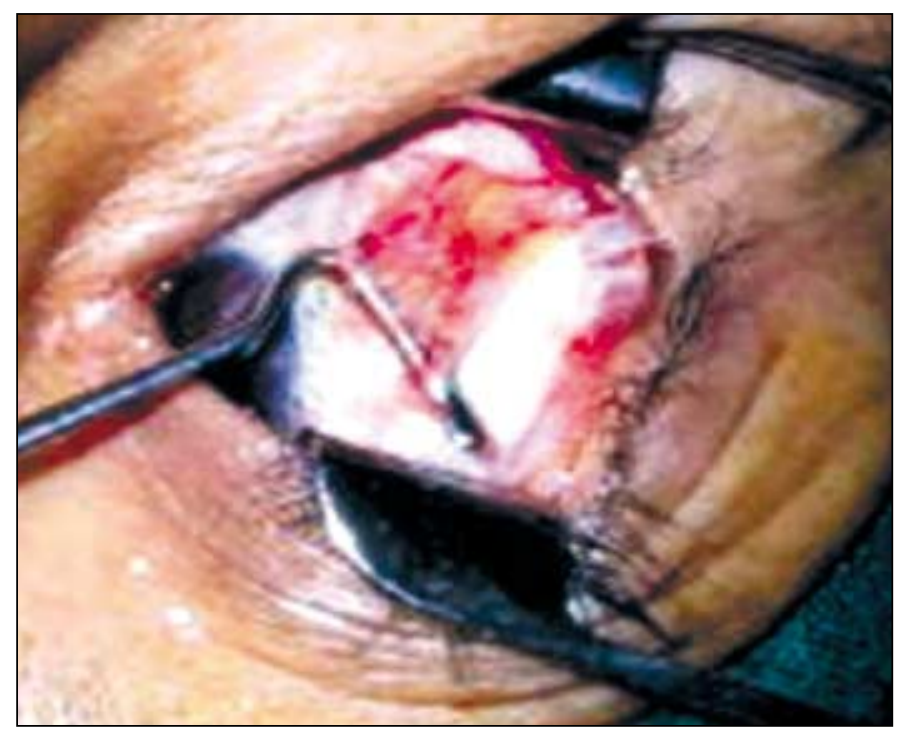

Figura 6 - Trajeto anômalo do músculo reto lateral

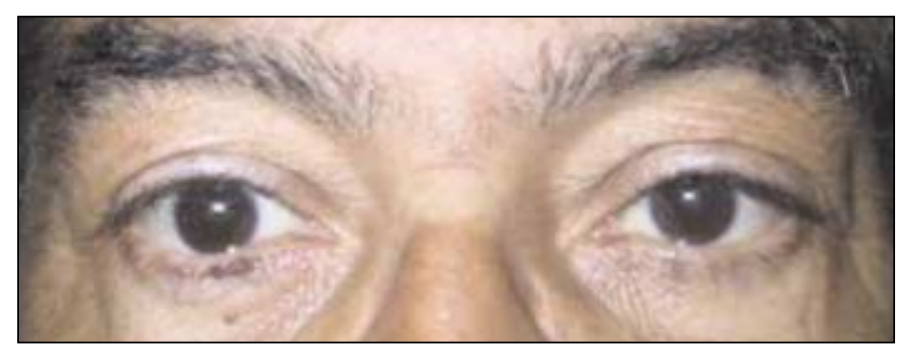

Figura 7 - Posição pós-operatória (PPO) 
sutura com fio absorvível $\left(\mathrm{Vycril}^{\circledR}\right.$ ), pois na descrição do trabalho original de Yamada, a sutura é feita com fio inabsorvível com o intuito de não ocorrer perda das fibras transpostas.

Sugerimos que para o sucesso da cirurgia as fibras transpostas devam estar com tônus adequado para atingirmos boa posição dos olhos no período pós-operatório tardio, como foi descrito por outros autores ${ }^{(12)}$ que descreveram, com o intuito de potencialização do efeito, a ressecção de fibras transpostas na técnica de Hummelsheim para estrabismos paralíticos. Na segunda abordagem cirúrgica do caso 1 , os feixes transpostos estavam frouxos, fato que poderia colaborar para o insucesso cirúrgico. Com a realização do pregueamento dos feixes transpostos durante a segunda abordagem, pudemos atingir bom resultado. Já no segundo caso com a ressecção de ambas as faixas antes de realizar a transposição, pudemos aumentar o seu tônus, não necessitando assim de outra cirurgia.

\section{ABSTRACT}

"Progressive esotropia fixus" is a disease present in high myopic patients with a large angle esotropia associated with hypotropia with poor surgical results. This paper has the purpose to describe Yamada's surgical technique (hemitranspositions of the superior rectus and lateral rectus) applied to 2 patients with good surgical results.
Keywords: Myopia/complications; Esotropia/etiology; Esotropia/surgery; Case report

\section{REFERÊNCIAS}

1. Bielschowsky A. Lectures on motor anomalies. Hanover: Dartmouth College; 1943. p.32-3.

2. Almeida H, Jampolsky A, Fitton M. Surgical results in esotropia associated with myopia. Am J Ophthalmol. 1965;60(3):448-52.

3. Costenbader FD. Infantile esotropia. Trans Am Ophthalmol Soc. 1961;59:397-429.

4. Demer J, Von Noorden GK. High myopia as an unusual cause of restrictive motility disturbance. Surv Ophthalmol. 1989;3(4):281-4.

5. Gamio S, Illescas N, Bollati M. Miopia y esotropia en la infância. Arch Oftalmol B Aires. 1993;68:111-7.

6. Knapp P. Surgical result of esotropia associated with myopia. In: $1^{\mathfrak{a}}$ Reunião dos Discípulos do Dr Ciancia, Foz do Iguaçu, 18 a 22 de maio de 1990. p.28-30.

7. Krzizok TH, Kaufmann H, Traupe H. New approach in strabismus surgery in high myopia. Br J Ophthalmol. 1997;81(8):625-30

8. Aoki Y, Nishida Y, Hayashi O, Nakamura J, Oda S, Yamade S, et al. Magnetic resonance imaging measurements of extraocular muscle path shift and posterior eyeball prolapse from the muscle cone in acquired esotropia with high myopia. Am J Ophthalmol. 2003;136(3)482-9.

9. Akizawa Y, Yasuzumi K, Tanaka A. [Morphological findings in progressive esotropia with high myopia]. Nippon Ganka Gakkai Zasshi. 2002;106(7):411-5. Japanese.

10. Herzau V, Ioannakis K. [Pathogenesis of eso- and hypotropia in high myopia]. Klin Monatsbl Augenheilkd. 1996;208(1):33-6. Germany.

11. Yamada M, Taniguchi S, Muroi T, Satofuka S, Nishina S. Rectus eye muscle paths after surgical correction of convergent strabismus fixus. Am J Ophthalmol. 2002;134(4):630-2.

12. Brooks SE, Olitsky SE, de B Ribeiro G. Augmented Hummelsheim procedure for paralytic strabismus. J Pediatr Ophthalmol Strabismus. 2000;37(4):189-95.

\section{ABO ELETRÔNICO}

\section{A versão eletrônica dos Arquivos Brasileiros de Oftalmologia com textos completos está disponível em:}

- ABO - Arquivos Brasileiros de 0 ftalmologia

http:/ / www.abonet.com.br

- SciElO - Scientific Electronic Library 0 nline

http:/ / www.scielo.org

- Free Medical J ournals - http:/ www.freemedicaljournals.com 